\title{
The Impact of Fluid Balances in the First 48 Hours on Mortality in the Critically III Patients
}

\author{
Azrina Md Ralib, Norhalini Hamzah, Majdiah Syahirah Nasir, and Mohd Basri Mat Nor" \\ Department of Anaesthesiology and Intensive Care, Kulliyyah (Faculty) of Medicine, International Islamic \\ University Malaysia
}

\begin{abstract}
Introduction: There has been increasing evidence of detrimental effects of cumulative positive fluid balance in critically ill patients. The postulated mechanism of harm is the development of interstitial oedema, with resultant increase morbidity and mortality. We aim to assess the impact of positive fluid balance within the first 48 hours on mortality in our local ICU population. Methods: This was a secondary analysis of a single centre, prospective observational study. All ICU patients more than 18 years were screened for inclusion in the study. Admission of less than 48 hours, post-elective surgery and ICU readmission were excluded. Cumulative fluid balance either as volume or percentage of body weight from admission was calculated over 6, 24 and 48 hour period from ICU admission. Results: A total of 143 patients were recruited, of these 33 died. There were higher cumulative fluid balances at 6, 24 and 48 hours in nonsurvivors compared to survivors. However, after adjusted for severity of illness, APACHE II Score, they were not predictive of mortality. Sensitivity analysis on sub-cohort of patients with acute kidney injury (AKI) showed only an actual 48-hour cumulative fluid balance was independently predictive of mortality (1.21 (1.03 to 1.42)). Conclusions: Cumulative fluid balance was not independently predictive of mortality in a heterogenous group of critically ill patients. However, in subcohort of patients with AKI, a 48-hour cumulative fluid balance was independently predictive of mortality. An additional tile is thus added to the mosaic of findings on the impact of fluid balance in a hetergenous group of critically ill patients, and in subcohort of AKI patients.
\end{abstract}

KEYWORDS: Fluid balance, acute kidney injury, Intensive Care Unit, mortality

\section{INTRODUCTION}

Fluid resuscitation is a major component of the acute management of critically ill patients. However, there has been increasing evidence of detrimental effects of cumulative positive fluid balance. $^{1-5}$ The postulated mechanism of harm is the development of interstitial oedema, with resultant increase morbidity and mortality. Interstitial oedema may result from aggressive fluid therapy to replace the non-existent 'third space' loss. In such cases there may be altered distribution of protein due to destruction of glycocalyx ${ }^{6}$ and altered Starling forces ${ }^{7}$ leading to the accumulation of fluid in the interstitial compartment. This results in impairment of the lung, cardiac, kidney and gastrointestinal function.

Several randomised controlled trials in surgical patients showed that positive fluid balance has been

Corresponding author:

Dr Azrina Md Ralib

Department of Anaesthesiology and Intensive

Care,

Kulliyyah (Faculty) of Medicine,

International Islamic University Malaysia,

Jalan Hospital Campus,

25000 Kuantan, Pahang.

Email:drazrina@gmail.com associated with higher morbidity and mortality. 2,5,8,9 However, the impact of fluid balance on critically ill patients are less being studied than in surgical patients. ${ }^{1,10,11}$ Alsous et al. showed that in septic shock patients, negative fluid balance independently predicted survival. ${ }^{11}$ Similarly, in a recent study on 137 sepsis patients, Acheampong \& Vincent showed an independent association between positive fluid balance on mortality. ${ }^{10}$. In contrast, Wiedemann et al. showed no difference in 60-day mortality between those with restrictive or liberal group in a large randomised controlled trial in patients with acute lung injury. ${ }^{1}$

Patients with acute kidney injury (AKI) are more prone to positive fluid balance due to impairment of kidney regulation of water balance. ${ }^{12,13}$ As kidney function declined in 253 AKI patients, fluid accumulation increased progressively. $^{14}$ Several studies had showed that positive fluid balance independently predicted mortality in AKI patients. $^{4,12,15-17}$

We aim to assess the impact of positive fluid balance within the first 48 hours of admission on mortality in our heterogenous group of critically ill patients. We also aim to evaluate the association in a sub-cohort of patients with AKI. 


\section{MATERIALS AND METHODS}

This was a secondary analysis of a single centre, prospective observational study of Hospital Tengku Ampuan Afzan, Kuantan. ${ }^{18}$ The study was registered under the Malaysian National Medical Research Register (NMRR-13-1631-18970, https://www.nmrr. gov.my). Ethical approval was obtained from the Malaysian Medical Ethics and Research Committee (MREC Number P14-663), and the International Islamic University Ethics Committee (IREC Number 266). All patients more than 18 years being admitted to ICU were reviewed. As only routinely available clinical information is collected, the need for informed consent is waived. Patients' clinical records and ICU charts were reviewed.

Hourly fluid input and output for the first 48 hours of admission were collected. Cumulative fluid balance as a percentage of body weight was calculated from ICU admission to 24 and 48 hours post-admission. Body weight was retrieved from the ICU charts as determined by the clinical staff from the most recent documented weight in the medical record, or as reported by the patient or relatives.

Daily plasma creatinine concentrations measured in the ICU were collected from the patients' clinical records. AKI was defined based on either creatinine or urine output criteria (AKl ${ }_{\text {uO }}$ ) of the KDIGO guideline. First creatinine measured on ICU admission was used as baseline creatinine. Plasma creatinine was analysed using the Olympus AU2700 ${ }^{T M}$ chemistry-immunoanalyser (Olympus, Philadelphia, USA). Other data that were collected includes age, sex, height, weight, ethnicity, clinical or surgical admission, and primary admission diagnosis, co-morbid diseases, duration of ICU and hospital stay, mechanical ventilation and its length, death and dialysis. The baseline Acute Physiology and Chronic Health Evaluation (APACHE) II and Sequential Organ Failure Assessment (SOFA) were used to assess severity of illness in each patients.

\section{Statistical Analysis}

Results are presented as mean \pm SD for normally distributed variables or median (inter-quartile range) for non-normally distributed variables. Comparison of variables between the two groups was analyzed using the independent $t$-test for normally distributed variables or the Mann-Whitney test for non-normally distributed variables. Categorical variables were compared with ChiSquare test for independent variables. Multivariable logistic regression was used to calculate odds ratios after adjusting for covariates. Variables were included as co-variates in regression analysis if they are at $p<0.1$ on univariate analysis. All OR, and HR are presented with $95 \%$ confidence intervals.

\section{RESULTS}

Three hundred and ten patients were screened between $21^{\text {st }}$ February 2014 to $3^{\text {rd }}$ May 2014. Patients less than 18 years $(n=20)$, with ICU admission of less than 48 hours $(n=112)$, post elective surgery $(n=27)$, and ICU readmission $(n=8)$ were excluded. After exclusion, a total of 143 patients were recruited in the study, of these $33(23 \%)$ died.

\section{Demographic and clinical profiles}

Patient demographic and clinical profiles between survivors and non-survivors are shown in Table I. Non -survivors had higher severity of illness compared to survivors, as depicted as higher SOFA and APACHE II scores $(p=0.001$ and $<0.0001$, respectively). There were no differences in other demographic and clinical profiles between survivors and non-survivors.

\section{Cummulative fluid balances}

Cumulative fluid balances, and percentage of fluid balance over body weight between survivors and non -survivors over 6, 24 and 48 hours are shown in Table II. There were higher cumulative fluid balances in non-survivors at 6,24 and 48 hours compared to survivors. Multivariate analyses for prediciton of mortality were shown in Table III.

Variable was added if they have a $p$ value of less than 0.1 on univariate analysis. Total SOFA and APACHE II scores had $p$ value less than 0.1 , however since they are linearly correlated, only APACHE II was added as co-variates. After adjusted for APACHE II Score, the fluid balances over 6,24 or 48 hours were not predictive of mortality.

We also investigated the association of fluid balances with length of mechanical ventilation and ICU stay on survivors $(n=110)$. There was a positive correlation between 48-hour cumulative fluid balance with length of ICU stay (Pearson correlation of $r=0.43, p<0.0001$ ), and with length of mechanical ventilation $(r=0.33, p=0.001)$.

\section{Sensitivity analysis: AKI patients}

Sixty-five patients $(n=93)$ had AKI. Patient with AKI had higher 48-hour cumulative fluid balance compared to those without AKI (Figure 1). In order to investigate the effect of positive fluid balance in AKI cohort, sensitivity analyses were performed in these patients. In these patients, a 48-hour actual cumulative fluid balance was independently predictive of mortality with an odds ratio of 1.21 (1.03 to 1.42). However, when the fluid balance was adjusted to body weight the association becomes smaller (OR of 1.09 (0.99 to 1.20) (Table IV). 
Table I: Demographic and clinical characteristics

\begin{tabular}{|c|c|c|c|c|}
\hline Variables & All patients & Non-survivors & Survivors & $\mathrm{p}$-value \\
\hline $\mathrm{n}$ & 143 & 33 & 110 & \\
\hline Age (years) & $50 \pm 16$ & $53 \pm 18$ & $50 \pm 16$ & 0.33 \\
\hline Ethnicity & & & & 0.54 \\
\hline Malay & $110(76.9)$ & $25(75.8)$ & $85(77.3)$ & \\
\hline Chinese & $17(11.9)$ & $4(12.1)$ & $13(11.8)$ & \\
\hline Indian & $4(2.8)$ & $1(3.0)$ & $3(2.7)$ & \\
\hline Orang Asli & 7 (4.9) & $3(9.1)$ & $4(3.6)$ & \\
\hline Others & $5(3.5)$ & $0(0)$ & $5(4.5)$ & \\
\hline Gender (Male) & $92(64.3)$ & $21(63.6)$ & $71(64.5)$ & 0.92 \\
\hline Weight (kg) & $67 \pm 15$ & $65 \pm 15$ & $68 \pm 15$ & 0.41 \\
\hline Height (Cm) & $159 \pm 17$ & $161 \pm 7$ & $162 \pm 8$ & 0.78 \\
\hline SOFA score & $7.7 \pm 3.7$ & $9.5 \pm 3.2$ & $7.2 \pm 3.7$ & 0.001 \\
\hline APACHE II score & $17.1 \pm 6.7$ & $21.8 \pm 6.4$ & $15.7 \pm 6.2$ & $<0.0001$ \\
\hline Sepsis & $79(55.2)$ & $22(66.7)$ & $57(51.8)$ & 0.13 \\
\hline Admission Category & & & & 0.29 \\
\hline Medical & $107(74.8)$ & $27(81.8)$ & $80(72.7)$ & \\
\hline Surgical & $36(25.2)$ & $6(18.2)$ & $30(27.3)$ & \\
\hline Primary Admission Category & & & & 0.10 \\
\hline Neurological & $16(11.2)$ & $0(0)$ & $16(14.5)$ & \\
\hline Respiratory & $53(37.1)$ & $13(39.4)$ & $40(36.4)$ & \\
\hline Infection & $19(13.3)$ & $5(15.2)$ & $14(12.7)$ & \\
\hline $\begin{array}{l}\text { Connective Tissue Dis- } \\
\text { ease/Autoimmune }\end{array}$ & $4(2.8)$ & $2(6.1)$ & $2(1.8)$ & \\
\hline Renal & $4(2.8)$ & $1(3.0)$ & $3(2.7)$ & \\
\hline Cardiovascular & $12(8.4)$ & $4(12.1)$ & $8(7.3)$ & \\
\hline $\begin{array}{l}\text { Gastrointestinal/ } \\
\text { Hepatobiliary/ Pancreas }\end{array}$ & $13(9.1)$ & $5(15.2)$ & $8(7.3)$ & \\
\hline Trauma & $20(14.0)$ & $2(16.4)$ & $18(16.4)$ & \\
\hline Endocrine/Metabolic & $1(0.7)$ & $0(0)$ & $1(0.9)$ & \\
\hline \multicolumn{5}{|l|}{ Comorbid Diseases } \\
\hline Hypertension & $52(36.4)$ & $13(39.4)$ & $39(35.5)$ & 0.68 \\
\hline Diabetes mellitus & $41(28.7)$ & $11(33.3)$ & $30(27.3)$ & 0.50 \\
\hline $\begin{array}{l}\text { Chronic cardiovascular } \\
\text { disease }\end{array}$ & $13(9.1)$ & $4(12.1)$ & $19(8.2)$ & 0.49 \\
\hline Chronic renal failure & $12(8.4)$ & $5(15.2)$ & $7(6.4)$ & 0.11 \\
\hline Chronic lung disease & $16(11.3)$ & $2(6.1)$ & $14(12.7)$ & 0.29 \\
\hline Chronic liver disease & $1(0.7)$ & $0(0)$ & $1(0.9)$ & 0.58 \\
\hline Immunosuppression & $3(2.1)$ & $3(9.1)$ & $0(0)$ & 0.001 \\
\hline Cancer & $1(0.7)$ & $0(0)$ & $1(0.9)$ & 0.58 \\
\hline Others & $11(7.7)$ & $9(27.3)$ & $47(42.7)$ & 0.11 \\
\hline
\end{tabular}

Data expressed as mean $\pm S D, n(\%)$, or median (lower quartile - upper quartile). APACHE II Score: Acute Physiological and Chronic Health Evaluation II Score. SOFA Score: Sequential Organ Failure Score.

Table II: Fluid balance in survivors and non-survivors at 6, 24 and 48 hours

\begin{tabular}{llll}
\hline Cumulative fluid balance & Non-survivors & Survivors & p-value \\
\hline $\mathrm{n}$ & 33 & 110 & 0.008 \\
6-hour & $1.8 \pm 2.5$ & $0.8 \pm 1.5$ & 0.01 \\
$\quad$ - Actual (L) & $2.5 \pm 3.5$ & $1.2 \pm 2.1$ & 0.001 \\
$\quad$ - Over BW (\%BW) & & & 0.006 \\
24-hour & $3.5 \pm 3.2$ & $1.9 \pm 2.2$ & $<0.0001$ \\
- Actual (L) & $4.8 \pm 4.8$ & $2.7 \pm 3.4$ & 0.002 \\
- Over BW (\%BW) & $4.9 \pm 4.3$ & $2.5 \pm 2.9$ & $3.7 \pm 4.3$ \\
48-hour & $6.8 \pm 6.8$ & & \\
- Actual (L) & & & \\
- Over BW (\%BW) & &
\end{tabular}

Data expressed as mean \pm SD. BW: Body Weight. Comparison between survivors and non-survivors were compared using independent $\mathrm{t}$-test 
Table III: Multivariate analysis for mortality

\begin{tabular}{lll}
\hline $\begin{array}{l}\text { Cumulative fluid bal- } \\
\text { ance }\end{array}$ & Mortality & p-value \\
\hline 6-hour & $1.17(0.92$ to 1.48$)$ & 0.19 \\
- Actual (L) & $1.13(0.96$ to 1.34$)$ & 0.14 \\
• Over BW (\%BW) & & \\
24-hour & $1.15(0.97$ to 1.37$)$ & 0.11 \\
- Actual (L) & $1.09(0.98$ to 1.21$)$ & 0.13 \\
- Over BW (\%BW) & & \\
48-hour & $1.14(0.99$ to 1.30$)$ & 0.06 \\
- Actual (L) & $1.07(0.98$ to 1.16$)$ & 0.12 \\
- Over BW (\%BW) & & \\
\end{tabular}

Adjusted for total APACHE II score. Variables were added separately. Data presented as OR and 95\% confidence interval.

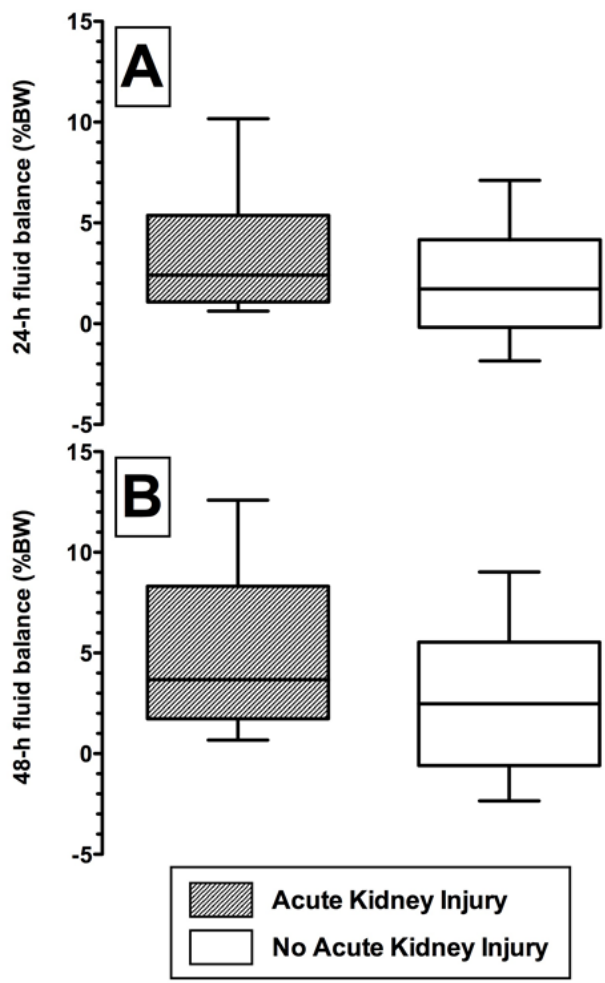

Figure 1: Cumulative fluid balance at 24 hours (A) and 48 hours (B) in patients with acute kidney injury versus no acute kidney injury. Independent t-test, $\mathrm{p}=(\mathrm{A}) 0.003$. and $(B)<0.0001$.

\section{DISCUSSION}

In this prospective study, we showed cumulative fluid balance at 6, 24 and 48 hours were higher in nonsurvivors compared to survivors. However, after adjusted for severity of illness the, cumulative fluid balance at 6, 24 or 48 hours were not associated with mortality. Cumulative fluid balance was not associated with mortality in a hetergenous group of critically ill patients. However, in subcohort of patients with AKI, a 48-hour cumulative fluid balance was independently predictive of mortality.
Table IV: Multivariate analysis for mortality in subcohort of patients with acute kidney injury (AKI)

\begin{tabular}{lll}
\hline $\begin{array}{l}\text { Cumulative } \\
\text { fluid balance }\end{array}$ & Mortality & p-value \\
\hline 6-hour & $1.31(0.94$ to 1.81$)$ & 0.11 \\
- Actual (L) & $1.21(0.98$ to 1.50$)$ & 0.08 \\
- Over BW (\%BW) & & \\
24-hour & $1.22(0.99$ to 1.49$)$ & 0.06 \\
- Actual (L) & $1.11(0.98$ to 1.25$)$ & 0.10 \\
- Over BW (\%BW) & \\
48-hour & $1.21(1.03$ to 1.42$)$ & 0.03 \\
- Actual (L) & $1.09(0.99$ to 1.20$)$ & 0.07 \\
- Over BW (\%BW) & \\
\hline
\end{tabular}

Adjusted for total APACHE II score. Variables were added separately. Data presented as OR and $95 \%$ confidence interval.

There were growing evidences from observational studies on the detrimental effects of positive fluid balance, with most on post surgical patients. Brandstrup $B$ et al. demonstrated that restrictive fluid strategy was associated with fewer complications in the postoperative period. ${ }^{9}$ Another two randomised controlled trials comparing restrictive and liberal fluid therapy in abdominal aorta aneurysm and colorectal surgical patients showed a lesser post-operative complications including, cardiopulmonary, thromboembolic, haemorrhagic, kidney, or wound infection or tissuehealing, and shorter hospital stay. ${ }^{2,8}$ In 479 post surgical patients, patients with intraoperative fluid balance more than 2 litres had higher ICU complications, and fluid balance was independently associated with mortality. ${ }^{5}$ In another study of 100 cardiovascular surgical patients, positive fluid balance occurred early in the intraoperative period and was associated with increased risk of AKI, longer ICU and hospital stays, and mechanical ventilation. ${ }^{3}$ However there was no difference in mortality.

Compared to surgical patients, the impact of fluid balance on critically ill patients are less being studied. Upadya et al.(2005), showed that in 87 critically ill patients, those with a negative fluid balance were twice more likely to be successfully weaned from mechanical ventilation than those with a positive fluid balance. ${ }^{19}$ In patients with acute lung injury, Wiedemann et al.(2006) showed in a large randomised controlled trial comparing fluid management strategies, the conservative group had better lung function resulting in decreased duration of mechanical ventilation and length of ICU stay compared to the liberal group (mean cumulative fluid balance of $6992 \mathrm{ml}$ ). ${ }^{1}$ In our study, we showed positive association between 48-hour cumulative fluid balance with length of mechanical ventilation and ICU stay in survivors.

Although positive fluid balance was associated with increased duration of mechanical ventilation and ICU stay, Wiedemann et al. showed no difference in 60- 
day mortality between those with restrictive or liberal group. ${ }^{1}$ Similar to this, we showed cumulative fluid balances over the first 48 hours of ICU admission were not independently associated with mortality. Alsous et al. showed that in 36 patients with septic shock, negative fluid balance of more than $-500 \mathrm{ml}$ for at least one day were three times more likely to survive. ${ }^{11}$ In a recent study on 173 sepsis patients, Acheampong \& Vincent showed that a positive fluid balance was independently associated with mortality. ${ }^{10}$ These differences maybe due to the population studied. Whilst, our study involved a more heterogenous group of critically ill patients, Wiedemann et al. involved patients with acute lung injury, and Alsous et al. and Acheampong et al. involved those with sepsis. An additional tile is thus added to the mosaic of findings on the impact of fluid balance in critically ill patients. Patients with severe sepsis have generalised capillary leaking resulting in sequestration of fluid in the interstitium, hence positive fluid balance may be a marker of inflammatory disease. Since sepsis is common in our population, further study restricting to only sepsis patients maybe of interest. A randomised controlled trial comparing standard, and conservative fluid balance in critically ill patient with severe sepsis may determine whether fluid balance directly results in higher morbidity or mortality.

AKI patients were more prone to have higher fluid balance. We showed that AKI patients had higher fluid balance compared to no AKI patients, consistent with other studies. ${ }^{12,13}$ Positive fluid balance in AKI is associated with poorer outcome. ${ }^{4,12,15-17,20}$ We showed similar finding, 48hour fluid balance was independently associated with mortality. In an analysis of 1120 patients with $\mathrm{AKI}$, mean fluid balance was an independent risk factor for 60-day mortality. ${ }^{12}$ A positive fluid balance greater than $10 \%$ of body weight, was associated with higher 60-day mortality amongst the 618 AKI patients. ${ }^{15}$ A post-hoc analysis of 306 patients with AKI demonstrated higher odds ratio for 60-day mortality in the liberal fluid management arm compared to the conservative group. ${ }^{16} \mathrm{~A}$ negative fluid balance was independently associated with lower hospital mortality. ${ }^{17}$ In 132 patients with AKI from a multicentre study of 10 Italian ICUs, fluid balance and urine output were independently predictive of mortality. ${ }^{4}$ The more severe AKI, the less likely it is to respond to the first bolus of fluid therapy. This may inappropriately prompt further fluid challenges, hence positive fluid balance may also reflect $A K I$ severity.

\section{Study limitations}

This study has several limitations. First, it was performed in only one centre and involved a small number of patients. A larger multicentre trial would provide a more robust data. Second, body weight was not measured, but determined from the most recent body weight documented in medical records, or reported by a patient or relative. However, there were no differences in body weight in univariate analysis of patients with and without positive fluid balance. Finally, we did not account for the type of fluid given in the study.

\section{CONCLUSION}

There were higher cumulative fluid balance in nonsurvivors compared to survivors, however, cumulative fluid balance was not independently predictive of mortality in a heterogenous group of critically ill patients. In subcohort of patients with AKI, a 48-hour cumulative fluid balance was independently predictive of mortality. An additional tile is thus added to the mosaic of findings on the impact of fluid balance in a hetergenous group of critically ill patients, and in sub-cohort of AKI patients.

\section{ACKNOWLEDGEMENT}

We would like to thank Sister Amimah Abdul Hamid, Siti Nabilah Zainuddin, Nurhafizah Zairudin and all nurses in the intensive care unit for their assistance in data collection for the study.

\section{REFERENCES}

1. Wiedemann HP, Wheeler AP, Bernard GR, et al. Comparison of two fluid-management strategies in acute lung injury. New Engl J Med 2006;354:2564-75.

2. McArdle GT, McAuley DF, McKinley A, et al. Preliminary results of a prospective randomized trial of restrictive versus standard fluid regime in elective open abdominal aortic aneurysm repair. Ann Sur 2009;250:28-34.

3. Kambhampati G, Ross EA, Alsabbagh MM, et al. Perioperative fluid balance and acute kidney injury. Clin Exp Nephrol 2012;16:730-738.

4. Teixeira C, Garzotto F, Piccinni P, et al. Fluid balance and urine volume are independent predictors of mortality in acute kidney injury. Crit Care 2013;17:R14.

5. Silva JM, Jr., de Oliveira AM, Nogueira FA, et al. The effect of excess fluid balance on the mortality rate of surgical patients: a multicenter prospective study. Crit Care 2013;17:R288.

6. Jacob M, Chappell D, Rehm M: The 'third space'-fact or fiction? Best Pract Res Clin Anaesth 2009, 23(2):145-57.

7. Schrier RW: Fluid administration in critically ill patients with acute kidney injury. Clin J Am Soc Nephrol 2010;5:733-9.

8. Khoo CK, Vickery CJ, Forsyth N, Vinall NS, EyreBrook IA: A prospective randomized controlled trial of multimodal perioperative management protocol in patients undergoing elective colorectal resection for cancer. Ann Surg 2007;245:867-2. 
9. Brandstrup B, Tønnesen $H$, Beier-Holgersen $\mathrm{R}$, et al. Effects of intravenous fluid restriction on postoperative complications: comparison of two perioperative fluid regimens: a randomized assessor-blinded multicenter trial. Ann Surg 2003; 238:641-8.

10. Acheampong A, Vincent JL. A positive fluid balance is an independent prognostic factor in patients with sepsis. Critical care 2015;19.

11. Alsous $F$, Khamiees $M$, DeGirolamo A, Amoateng-Adjepong Y, Manthous CA: Negative fluid balance predicts survival in patients with septic shock: a retrospective pilot study. Chest 2000;117:1749-54.

12. Payen D, de Pont AC, Sakr Y, Spies C, Reinhart $\mathrm{K}$, Vincent JL: A positive fluid balance is associated with a worse outcome in patients with acute renal failure. Crit Care 2008;12:R74.

13. Van Biesen W, Yegenaga I, Vanholder R, et al. Relationship between fluid status and its management on acute renal failure (ARF) in intensive care unit (ICU) patients with sepsis: a prospective analysis. J Nephrol 2005;18:54-60.

14. Macedo E, Bouchard J, Soroko SH, et al. Fluid accumulation, recognition and staging of acute kidney injury in critically-ill patients. Crit Care 2010;14:R82.

15. Bouchard J, Soroko SB, Chertow GM, et al. Fluid accumulation, survival and recovery of kidney function in critically ill patients with acute kidney injury. Kidney Int 2009;76:422-7.

16. Grams ME, Estrella MM, Coresh J, Brower RG, Liu KD: Fluid balance, diuretic use, and mortality in acute kidney injury. Clin J Am Soc Nephrol 2011;6:966-73.

17. The RENAL Replacement Therapy Study Investigators: An observational study fluid balance and patient outcomes in the randomized evaluation of normal vs. augmented level of replacement therapy trial. Crit Care Med 2012;40:1753-60.

18. Md Ralib A, Mat Nor MB: Acute kidney injury in a Malaysian intensive care unit: Assessment of incidence, risk factors, and outcome. J Crit Care 2015;30:636-42.

19. Upadya A, Tilluckdharry L, Muralidharan V, Amoateng-Adjepong $\mathrm{Y}$, Manthous CA: Fluid balance and weaning outcomes. Intens Care Med 2005;31:1643-47.

20. Vaara ST, Korhonen AM, Kaukonen KM, et al. Fluid overload is associated with an increased risk for 90-day mortality in critically ill patients with renal replacement therapy: data from the prospective FINNAKI study. Crit Care 2012;16:R197. 